\title{
"CRITERIOS DE DISEÑO PARA LA TOMA DE DECISIONES EN LA EVALUACIÓN DE ALTERNATIVAS DE MINADO EN EMPRESAS MINERAS DE LA REGIÓN CENTRAL DEL PERÚ"
}

\section{"DESIGN CRITERIA FOR DECISION MAKING IN THE EVALUATION OF MINING ALTERNATIVES IN COMPANIES IN CENTRAL REGION OF PERU"}

\author{
Mendiola Ochante, Victor Javier ${ }^{1}$
}

\section{RESUMEN}

El diseño de una mina tiene un conjunto de procesos para alcanzar sus objetivos estratégicos y su visión entre Ios que podemos destacar: la selección del método de minado, el dimensionamiento geométrico de la mina, la determinación del ritmo anual de producción y la ley de corte, la secuencia de extracción, etc. La presente investigación fijo como problema principal, ¿Cuáles son los criterios de diseño empleados en la selección de métodos de minado en las empresas mineras de la región central del Perú?; como objetivo general: "Determinar los criterios de diseño; para la selección de métodos de minado en las empresas mineras de la región central del Perú" y como hipótesis general: "Los criterios de diseño utilizados en la selección de métodos de minado en las empresas mineras de la región central del Perú, no son óptimos". Se utilizó el método no experimental, transeccional descriptivo. El diseño empleado fue descriptivo simple y correlacional. Concluyendo que; solo el 16.33\%, de las empresas mineras cumplen parcialmente con los criterios de diseño para la selección de métodos de minado. Por consiguiente se valida la hipótesis planteada en nuestra investigación. Siendo estas empresas las siguientes: Compañía minera San Ignacio de Morococha, unidad San Vicente (Mediana minería); Volcán Compañía Minera S.A.A. (Gran minería); Minera Argentum S.A., Morococha (Mediana minería). Respecto a la toma de decisiones de las empresas seleccionadas podemos concluir que solo el 5\%, realiza una simulación y cuenta con un proceso de diseño capaz de analizar alternativas multicriterio. Seleccionar el método de minado optimó extractivo es uno de los problemas de decisión multicriterio (DM) en el cual los decisores deben asignar un peso a los criterios para realizar una simulación. Para resolver este problema se plantea la ponderación de variables mediante pesos y la selección del método mediante la sumatoria de los mismos.

\section{Palabras Clave}

Análisis de decisión multicriterio, selección del método de explotación minera, criterios de diseño.

\section{ABSTRACT}

The design of a mine has a group of processes to reach its strategic objectives and its vision among those that we can highlight: the selection of the method of minning, the geometric dimensionamiento of the mine, the determination of the annual rhythm of production and the court law, the extraction sequence, etc. Does the present investigation fix as main problem, Which are the approaches of design employees in the selection of methods of having mined in the mining companies of the central region of the Peru?; as general objective: "To determine the design approaches; for the selection of methods of having mined in the mining companies of the central region of the Peru" and I eat general hypothesis: "The design approaches used in the selection of methods of having mined in the mining companies of the central region of the Peru, are not good". You uses the non experimental method, descriptive transeccional. The used design was descriptive simple and correlacional. Concluding that; alone $16.33 \%$, of the mining companies fulfills the design approaches partially for the selection of methods of 1 Investigador : Docente, Administrativo, Estudiante / Sin vinculo 
having mined. Consequently the hypothesis outlined in our investigation been been worth. Being these companies the following ones: Mining company San Ignacio of Morococha, unit San Vicente (Medium mining); Volcano Mining Company S.A.A. (Great mining); Mining Argentum CORP., Morococha (Medium mining). Regarding the taking of decisions of the selected companies can conclude that alone $5 \%$, he/she carries out a simulation and bill with a design process able to analyze alternative multicriterio. To select the method of having mined extractive optimó is one of the problems of decision multicriterio (DM) in which the decisores should assign a weight to the approaches to carry out a simulation. To solve this problem he/she thinks about the ponderación of variables by means of pesos and the selection of the method by means of the sumatoria of the same ones.

Keywords: Decision Analysis multiopinion, designing, selection of the mining operation, .

\section{INTRODUCCIÓN}

Una explotación minera puede realizarse en superficie 0 debajo de ella, dependiendo de la profundidad del yacimiento y de otros parámetros técnicos, por 10 cual las extracciones se clasifican principalmente en minería a cielo abierto y minería subterránea, cada una con diferentes métodos correspondientes a unos parámetros particulares; los métodos subterráneos se emplean cuando la profundidad del yacimiento es excesiva para llegar por explotación a cielo abierto. La gran cantidad de criterios que pueden considerarse para seleccionar un método extractivo hace de esta una decisión bastante compleja para el decisor.

La selección de métodos extractivos en la minería es uno de los problemas de selección más antiguos de la humanidad, por tratarse de una actividad que tiene miles de años; la literatura científica más relevante al respecto comienza con Boshkov y Wright (1973), quienes plantean uno de los primeros esquemas cualitativos de clasificación para seleccionar los métodos extractivos. Un par de años después Morrison (1976), propone un sistema de clasificación el cual divide la minería subterránea en tres grupos basado en las condiciones del terreno asignando a cada uno el tipo de soporte requerido. Laubsher (1981), propone una metodología de selección para el método de extracción subterráneo basada en el sistema de clasificación R.M.R por sus siglas en inglés (rock mass raiting). La primera aproximación a un método de selección cuantitativo se da en el año de 1981cuando David E. Nicholas formula una aproximación numérica para la selección de método extractivo con su trabajo "Selection Procedure - A Numerical Approach" el cual formula el uso de una escala para la ponderación de cada uno de los métodos extractivos. Hartman (1987) desarrolla un esquema de selección basado en la geometría del yacimiento y las condiciones del terreno para escoger el método extractivo.

Posteriormente Miller-Tait, L.,Panalkis, R., Poulin, R., (1995), de universidad de British Columbia modifican el método Nicholas y agregan nuevos valores a la escala. Finalmente en la actualidad existen algunos abordajes al problema de selección del método de explotación, mediante análisis de decisión multicriterio entre las cuales se destaca la aplicación de lógica difusa en los trabajos de Bitarafan, M.R., Ataei, M. (2004), y también en Karadogan, A., Kahriman, A., \& Ozer, U (2008); otro método de decisión multicriterio utilizado para resolver este problema ha sido AHP (Analitic hierarchy proces) o PAJ (análisis de procesos jerárquicos); aplicado en los trabajos de Alpay, S., \& Yavuz, M. (2009), Azadeh, 
A., Osanloo, M., \& Ataei, M. (2010), y Bogdanovic, D., Nikolic, D., \&lvana, I. (2012). Una revisión de los principales métodos multicriterio puede consultarse en Figueira, J., Greco, S., and Ehrgott, M., y Barba Romero. En el presente trabajo se ha empleado el método de asignación de peso mediante un proceso de toma de decisiones junto con el método para la decisión multicriterio. Queriendo plantear un problema de decisión basado en información cuantitativa el cual puede ser evaluado mediante el criterio de expertos.

Para resolver este problema se genera una propuesta metodológica la cual consta de las siguientes etapas: 1. Definir el problema 2. Análisis del problema, 3. Búsqueda de alternativas de solución, 4. Decisión en función a criterios de diseño, 5. Especificación de la solución final.

La presente investigación fijo como problema principal, ¿Cuáles son los criterios de diseño empleados en la selección de métodos de minado en las empresas mineras de la región central del Perú?; como objetivo general: Determinar los criterios de diseño; para la selección de métodos de minado en las empresas mineras de la región central del Perú y como hipótesis general: Los criterios de diseño utilizados en la selección de métodos de minado en las empresas mineras de la región central del Perú, no son óptimos.

Se utilizó el método no experimental, transeccional descriptivo. El diseño fue el Diseño descriptivo simple y diseño descriptivo correlacional. Concluyendo que los criterios que deben cumplir las empresas mineras durante la selección de sus métodos de minado, se determinó que solo el 16.33\%, cumplen parcialmente con este modelo de selección de métodos de minado. Por consiguiente se valida la hipótesis planteada en nuestra investigación. Siendo estas empresas las siguientes: Compañía minera San Ignacio de Morococha, unidad San Vicente (Mediana minería); Volcán Compañía Minera S.A.A, (Gran minería); Minera Argentum S.A., Morococha (Mediana minería);

Respecto a la toma de decisiones de las empresas seleccionadas podemos concluir que solo el 5\%, realiza una simulación y cuenta con un proceso de diseño capaz de analizar alternativas.

\section{MATERIAL Y METODOS}

\section{METODOLOGIA DE INVESTIGACION:}

\section{a) Variables}

- Métodos numéricos

- Perspectivas financieras

- Perspectivas cliente

- Perspectivas internas

- Perspectiva aprendizaje

- Eficiencia

- Calidad

- Recursos humanos

- Criterios de diseño

- Seguridad y salud ocupacional

\section{b) Método de investigación}

Se utilizó el método no experimental, transeccional descriptivo. Ya que se describió e interpreto un conjunto de hechos en su estado actual y objetivo referente a la aplicación de metodología de métodos de minado en las empresas mineras subterráneas de la región central.

\section{c) Diseño}

Los diseños de investigación que se empleó fueron:

- Diseño descriptivo simple, se buscó y recopilo datos acerca de la metodología de selección de métodos de minado. El esquema fue el siguiente:

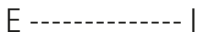

Dónde:

E: Empresa minera de la región central.

I: es la información sobre la selección de métodos de minado.

- Diseño descriptivo correlacional, con el propósito de investigar dos o más variables de la selección del método de minado en las empresas mineras de la región central, tratando de establecer relaciones entre ellas

\section{- Procesamiento y análisis de datos}

Se realizó el procesamiento de datos reportados en el campo, para luego realizar el análisis e interpretación de los mismos. 


\section{d) Población y muestra}

La muestra del estudio estuvo constituida por la población objeto de estudio: 49 empresas mineras con minado subterráneo de la región central.

\section{e) Técnicas y procedimientos de recolección de datos}

- Técnicas:

a. La observación participante.

b. La encuesta a gerentes de operaciones, gerentes de línea de las unidades mineras.

c. La entrevista a informantes previamente seleccionados.

- Instrumentos:

a. Guía de observación participante estructurada.

b. Cuestionario o cédula de encuesta.

c. Entrevistas estructuradas a informantes claves.

\section{- Fuentes de información y documentales}

a. Fuentes directas: datos empíricos directamente recopilados de los actores a través de las técnicas e instrumentos ya diseñados.

b. Fuentes indirectas: datos operativos de las empresas mineras.

\section{f) Técnicas de procesamiento y análisis de datos}

Se utilizó la estadística descriptiva, tablas, gráficos, etc.

\section{g) Materiales y equipos}

Los materiales que se utilizó fueron: estadísticas obtenidas por la UNCP, informes operacionales y de procesos de la empresas mineras y bibliografías relacionadas al respecto, etc.

Respecto a los equipos se utilizaron: Computadora, impresora, grabadoras, etc.

\section{RESULTADOS Y DISCUSIÓN}

Para validar las hipótesis planteadas se analizó los siguientes resultados de las encuestas tomadas a las 49 empresas mineras de la región central que están minando sus yacimientos por métodos subterráneos, siendo las principales:
- Cía. Minera Volcán S.A.A.

Método de explotación

costo de minado

Over Cut and Fill:

16.5 \$/ton.

Sub Level Caving: $\quad 9.5$ \$/ton.

Block Caving

10.5 \$/ton.

Room and Pillar Mining:

18.3 \$/ton.

Bench and Fill stoping

Cut and Fill Stoping

Shrinkage Stoping

Under Cut and Fill

Sub level stoping

- Cia. Minera Argentum S.A.

Sub level stoping.

Corte y relleno ascendente.

Bench and Fill stoping

Cut and Fill Stoping

Shrinkage Stoping

- Cía. Minera San Ignacio de Morococha S.A. Cut and Fill Stoping

\section{Validación de la Hipótesis general}

$\mathrm{Ha}=$ "Los criterios de diseño utilizados en la selección de métodos de minado en las empresas mineras de la región central del Perú, no son óptimos".

\section{Evaluación para definir el método de minado factible a ser aplicado.}

Para determinar la factibilidad del método de minado a aplicar en un cuerpo mineralizado, con las propiedades requeridas para la aplicación de dicho método de minado. De esta forma el método o los métodos que mejor se ajusten, serán considerados técnicamente factibles y podrán ser evaluados económicamente.

Las técnicas de selección para evaluar el método de minado considera generalmente dos aspectos fundamentales:

- Las características físicas y geológicas del cuerpo mineralizado, y

- Las condiciones geotécnicas del cuerpo mineralizado y de sus rocas encajonantes.

Para la selección del método o métodos de minado se puede aplicar la técnica propuesta por David E. 
Nicholas (1981) la cual se basa en definir un puntaje a los métodos de explotación considerados. Aquellos métodos que presentan un mayor puntaje, presentarán las condiciones mas favorables para su aplicación. La tabla abajo mostrada es un ejemplo de un ranking elaborado en base a la aplicación de la técnica de Nicholas a un caso específico.

Adicionalmente a este proceso las dimensiones y geometrías de explotación deben ser evaluadas geotécnicamente con el Método Gráfico de Estabilidad de Laubscher (1990) a fin de establecer los radios hidráulicos que eviten la activación de un potencial derrumbe 0 inestabilidad de un macizo rocoso.

\begin{tabular}{|l|c|c|c|}
\hline \multicolumn{1}{|c|}{ Método } & $\begin{array}{c}\text { Geometría del cuerpo y } \\
\text { distribución de leyes }\end{array}$ & $\begin{array}{c}\text { Conducción } \\
\text { geotécnica }\end{array}$ & $\begin{array}{c}\text { Puntaje } \\
\text { total }\end{array}$ \\
\hline $\begin{array}{l}\text { SUBLEVEL } \\
\text { CAVING (SLC) }\end{array}$ & 16 & 25 & 41 \\
\hline $\begin{array}{l}\text { SHINKAGE } \\
\text { STOPING (SKS) }\end{array}$ & 13 & 25 & 38 \\
\hline $\begin{array}{l}\text { BLOCK CAVING } \\
\text { (BC) }\end{array}$ & 12 & 25 & 37 \\
\hline $\begin{array}{l}\text { CUT \& FILL STO- } \\
\text { PING (C\&FS) }\end{array}$ & 11 & 26 & 37 \\
\hline $\begin{array}{l}\text { ROOM \& PILLAR } \\
\text { (R\&P) }\end{array}$ & -40 & 15 & -25 \\
\hline $\begin{array}{l}\text { SUBLEVEL OPEN } \\
\text { STOPING (SLOS) }\end{array}$ & 13 & -40 & -27 \\
\hline LONGWALL & -90 & 20 & -70 \\
\hline
\end{tabular}

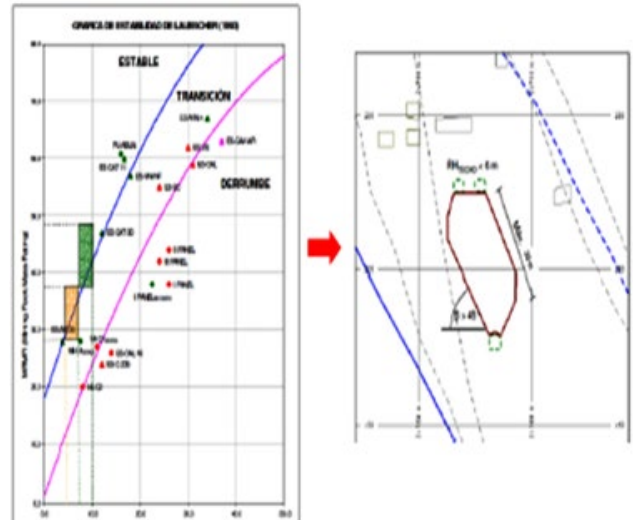

- El estudio de la posibilidad de elección de un determinado método de explotación comprende varias fases cuyo objetivo final es diseñar un sistema de extracción apropiado bajo las características del yacimiento. Esto puede ser interpretado como el camino para alcanzar la máxima utilidad en la operación.
- Hoy en día para abrir una mina o para cambiar el método de explotación, es de suma importancia ejecutar un proceso de selección del método de explotación mediante un análisis sistemático, global y específico del yacimiento

Los factores que tienen un mayor peso en la primera etapa de elección son:

- Geometría del yacimiento.

- Distribución de leyes.

- Propiedades geomecánicas del mineral y de las cajas.

Se refiere a las características geológicas del mineral y roca encajante. La investigación geológica debe permitir facilitar información relativa a:

- Correcta evaluación de reservas con las que cuenta el depósito.

- Principales tipos de roca.

- Zonas de alteración superficial e interna.

- Principales estructuras y accidentes tectónicos.

La geometría del yacimiento se define a través de su forma general, potencia, inclinación y profundidad.

- La distribución de leyes se define como:

- Uniforme

- Gradual

- Diseminada

- Errática

Analizado los criterios que deben cumplir las empresas mineras durante la selección de sus métodos de minado se determinó que solo el $16.33 \%$, cumplen parcialmente con este modelo de selección de métodos de minado. Por consiguiente se valida la hipótesis planteada en nuestra investigación. Siendo estas empresas las siguientes:

\begin{tabular}{|c|c|c|c|}
\hline Orden & Unidad & Clas. & $\begin{array}{c}\text { Empresa } \\
\text { CIA.MRA.SAN IGNACIO DE MORO- } \\
\text { COCHA SA. }\end{array}$ \\
\hline 2 & TICLIO & GM & A.MINERA S.A.A. \\
\hline 3 & MOROCOCHA & MM & MINERA ARGENTUM S.A. \\
\hline 4 & MANUELITA & MM & MINERA ARGENTUM S.A. \\
\hline 5 & ANTICONA & MM & MINERA ARGENTUM S.A. \\
\hline 6 & CARAHUACRA & GM & VOLCAN CIA.MINERA S.A.A. \\
\hline 7 & SAN CRISTOBAL & GM & VOLCAN CIA.MINERA S.A.A. \\
\hline 8 & ANDAYCHAGUA & GM & VOLCAN CIA.MINERA S.A.A. \\
\hline
\end{tabular}




\section{Validación de la Hipótesis específica}

$\mathrm{Ha}=$ "Los criterios de diseño necesarios para una toma de decisiones óptima incluyen: el modelo numérico, económico, ritmos de producción, disponibilidad de recursos humanos, seguridad y salud ocupacional".

La clasificación propuesta por Nicholas determina un método de explotación factible por medio de un ranking numérico y es verdaderamente cuantitativo. El primer paso es para clasificar la geometría del mineral y la distribución de leyes. Las características mecánicas de la roca de la zona mineralizada, el techo o muro colgante, el piso o muro yacente, son igualmente clasificadas. Así entonces los valores de los resultados que arroja este ejercicio se entienden de la siguiente manera:

- Un valor de 1 y 2 indica que una característica es probablemente adecuada a este método.

- Un valor de 3 y 4 indica que las características son preferenciales para el método de explotación.

- Un valor de 0 indica que esta característica no promueve el uso de este método de explotación.
- $\quad$ Y un valor de -49 indica que no se considerara indudablemente este método de explotación.

Por consiguiente para cumplir esta hipótesis debemos simular todos los criterios necesarios para determinar el método optimo que nos permitiría tener una empresa optima y competitiva, analizando la toma de decisiones de las empresas seleccionadas podemos concluir que solo el $5 \%$, realiza una simulación y cuenta con un proceso de diseño capaz de analizar alternativas. Ya que para obtener calidad en el diseño se tiene que simular cada proceso que participa en la obtención de la producción requerida en el minado del yacimiento.

Es decir ninguna de las empresas analizadas cumple con un análisis como a continuación mostramos para seleccionar la mejor alternativa de minado donde se presenta una simulación con 21 criterios, para la selección de una alternativa de minado:

ALTERNATIVAS

OBJETIVOS

SUBNIVEL

CONTRAPOZOS Y CORTE A SUBNIVEL

\begin{tabular}{|c|c|c|c|c|c|c|c|}
\hline \multirow[b]{2}{*}{ OBLIGATORIOS } & & & \\
\hline & & \multicolumn{2}{|l|}{ INFORMACIÓN } & $\begin{array}{l}\text { PASA/NO } \\
\text { PASA }\end{array}$ & \multicolumn{2}{|l|}{ INFORMACIÓN } & $\begin{array}{l}\text { PASA/NO } \\
\text { PASA }\end{array}$ \\
\hline \multicolumn{2}{|l|}{ Cumplir con las fechas programadas } & \multicolumn{2}{|c|}{ Dentro del programa actual } & PASA & \multicolumn{2}{|c|}{$\begin{array}{l}\text { Programa alternativo, pero } \\
\text { necesitaría algunas tone- } \\
\text { ladas del bloque } 2 G / 2 B\end{array}$} & PASA \\
\hline \multicolumn{2}{|l|}{ Conservar la estabilidad del subnivel $18 \mathrm{c}$} & \multicolumn{2}{|l|}{ Ref. JPD/OPS MIN 4.2} & PASA & \multicolumn{2}{|l|}{ Ref. SBL/RCM MIN 4.2} & PASA \\
\hline Que sea rentable & & $\begin{array}{l}\text { Referencia memo Jt/2. } \\
\text { MIN } 8.10 .2 \text { de } \\
\text { Noviembre lo. }\end{array}$ & & PASA & $\begin{array}{l}\text { Referencia memo } \\
\text { FoS } / 2.1 / \text { MIN } \\
8.10 .3 \text { de Octubre } 29\end{array}$ & & PASA \\
\hline DESEADOS & PESO & INFORMACIÓN & PUNTAJE & $\begin{array}{c}\text { PUNTAJE } \\
\text { PONDE- } \\
\text { RADO }\end{array}$ & INFORMACIÓN & PUNTAJE & $\begin{array}{c}\text { PUNTAJE } \\
\text { PONDE- } \\
\text { RADO }\end{array}$ \\
\hline Recuperación máxima de metal & 10 & 186,000 toneladas & 10 & 100 & 177,000 toneladas & 9 & 90 \\
\hline Costo mínimo/metal por tonelada & 10 & \$288/tonelada & 7 & 70 & 177,000 toneladas & 10 & 100 \\
\hline $\begin{array}{l}\text { Proporcionar condiciones seguras } \\
\text { para el personal de operación }\end{array}$ & 10 & $\begin{array}{l}\text { El menor riesgo para } \\
\text { los hombres }\end{array}$ & 10 & 100 & Riesgo moderado & 7 & 70 \\
\hline
\end{tabular}




\begin{tabular}{|c|c|c|c|c|c|c|c|}
\hline $\begin{array}{l}\text { Confianza en el éxito tomando en } \\
\text { cuenta el relleno y la estabilidad } \\
\text { de las estructuras }\end{array}$ & 10 & $\begin{array}{l}\text { El menor apoyo en el } \\
\text { relleno y la estabilidad } \\
\text { de las estructuras }\end{array}$ & 10 & 100 & $\begin{array}{l}\text { Cierto apoyo en el } \\
\text { relleno y la estabilidad }\end{array}$ & 5 & 50 \\
\hline Buenas condiciones de ventilación & 8 & Ningún problema & 10 & 80 & $\begin{array}{l}\text { Necesidad de llevar aire } \\
\text { puro a la cabeza de la } \\
\text { labor a través de los } \\
\text { pilares y contrapozos }\end{array}$ & 7 & 56 \\
\hline Máxima flexibilidad de la producción & 8 & $\begin{array}{l}\text { Se requieren muchos } \\
\text { puntos de carga }\end{array}$ & 10 & 80 & $\begin{array}{l}\text { Se necesitan pocos } \\
\text { puntos de carga }\end{array}$ & 7 & 56 \\
\hline $\begin{array}{l}\text { Diferir el avance hacia el sur de las } \\
\text { labore primarias }\end{array}$ & 8 & Ningún problema & 10 & 70 & $\begin{array}{l}\text { Requeriría de bancadas } \\
\text { primarias antes de usar } \\
\text { la cueva a subnivel }\end{array}$ & 2 & 14 \\
\hline
\end{tabular}

OBJETIVOS

CORTE A SUBNIVEL

ALTERNATIVAS

\begin{tabular}{|c|c|c|c|c|c|c|c|}
\hline \multirow[b]{2}{*}{ DESEADOS } & \multicolumn{4}{|c|}{ CORTE A SUBNIVEL } & \multicolumn{3}{|c|}{ CONTRAPOZOS Y CORTE A SUBNIVEL } \\
\hline & PESO & INFORMACIÓN & PUNTAJE & $\begin{array}{l}\text { PUNTAJE } \\
\text { PONDE- } \\
\text { RADO }\end{array}$ & INFORMACIÓN & PUNTAJE & $\begin{array}{l}\text { PUNTAJE } \\
\text { PONDE- } \\
\text { RADO }\end{array}$ \\
\hline $\begin{array}{l}\text { Asegurar la sencillez } \\
\text { de la operación }\end{array}$ & 5 & $\begin{array}{l}\text { Operación sencilla y } \\
\text { repetida }\end{array}$ & 10 & 50 & Algunas complicaciones & 7 & 35 \\
\hline $\begin{array}{l}\text { Un mínimo desarrollo } \\
\text { en el rellenado }\end{array}$ & 5 & $\begin{array}{l}\text { No es necesario un } \\
\text { desarrollo }\end{array}$ & 10 & 50 & $\begin{array}{l}4000 \text { pies de desarrollo } \\
\text { en el rellenado }\end{array}$ & 1 & 5 \\
\hline $\begin{array}{l}\text { Un mínimo de piedras de } \\
\text { azolve en el flujo de mineral }\end{array}$ & 5 & $\begin{array}{l}\text { Tenderia a haber más } \\
\text { debido a la dilución } \\
\text { dentro de la cueva }\end{array}$ & 8 & 40 & Mínimo & 10 & 50 \\
\hline Requisito mínimo de gente & 4 & $\begin{array}{l}\text { Algunos requerimientos } \\
\text { de gente extra }\end{array}$ & 8 & 32 & $\begin{array}{l}\text { Se esperarían más tonela- } \\
\text { das/turno-hombre }\end{array}$ & 10 & 40 \\
\hline Mínima extensión de desarrollo & 4 & $\begin{array}{l}\text { Consultar el memorándum } \\
\text { JL/2.1/MIN 8.10.2 }\end{array}$ & 8 & 32 & $\begin{array}{l}\text { Consultar el memorándum } \\
\text { FoS/2.1/MIN 8.10.3 }\end{array}$ & 10 & 40 \\
\hline Minimas necesidades de equipo & 4 & $\begin{array}{l}\text { Se necesitaría más esquioi } \\
\text { de perforación }\end{array}$ & 9 & 36 & Mínimo & 10 & 40 \\
\hline $\begin{array}{l}\text { Utilización máxima del } \\
\text { equipo disponible }\end{array}$ & 3 & $\begin{array}{l}\text { Un mínimo de requeri- } \\
\text { mientos especiales }\end{array}$ & 10 & 30 & $\begin{array}{l}\text { Se necesitarían pequeñas unidades } \\
\text { de carga entre en desarrollo y el } \\
\text { rellenado y también podría } \\
\text { necesitarse equipo de apoyo especial }\end{array}$ & 8 & 24 \\
\hline $\begin{array}{l}\text { Con la menor cantidad } \\
\text { de agua ocasional }\end{array}$ & 2 & $\begin{array}{l}\text { El agua se drenará y no } \\
\text { estará necesariamente en } \\
\text { los subniveles de producción }\end{array}$ & 10 & 20 & $\begin{array}{l}\text { Mas agua que sacar } \\
\text { como en el caso de las } \\
\text { labores a cielo abierto }\end{array}$ & 7 & 14 \\
\hline Con los mejores accesos & 2 & Buenos caminos de acceso & 10 & 20 & $\begin{array}{l}\text { El relleno proporciona } \\
\text { malos caminos de accesos } \\
\text { al afectarse con el agua }\end{array}$ & 8 & 16 \\
\hline $\begin{array}{l}\text { Un mínimo de cemento } \\
\text { en el flujo demineral }\end{array}$ & 1 & Un mínimo de cemento & 10 & 10 & $\begin{array}{l}\text { Más extracción a través } \\
\text { del cemento }\end{array}$ & 8 & 8 \\
\hline TOTAL & & & & 976 & & & 788 \\
\hline
\end{tabular}




\section{FASES DEL PROCESO DE DECISIÓN MULTICRITERIO PLANTEADO POR EL AUTOR}

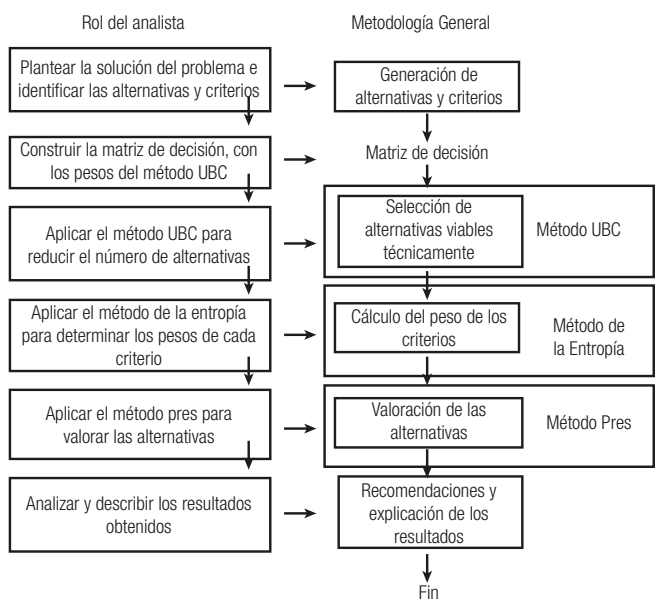

Determinar la alternativa de minado solo teniendo en cuenta los criterios de Hartman y Nicolás, solo es el primer paso para responder al problema de encontrar la alternativa óptima.

En problemas de selección los cuales cuenten con gran número de criterios se tiene que trabajar con expertos, si seguimos la metodología propuesta generaremos en forma sencilla una forma acertada de encontrar la mejor alternativa de minado que requiere el yacimiento.

\section{CONCLUSIONES}

1. Los factores que tienen un mayor peso en la primera etapa de selección del método minero son los relativos a la geometría y distribución de leyes del depósito, y a las propiedades geomecánicas del mineral y estériles adyacentes. En la segunda etapa se procede a la evaluación económica, basada sobre un esquema general de explotación, así como al estudio complementario del ritmo de producción y de la ley de corte, necesidades de personal, impactos ambientales y procedimientos de restauración y otras consideraciones específicas.

2. Hoy en día para abrir una mina o para cambiar el método de explotación, es de suma importancia ejecutar un proceso de selección del método de explotación mediante un análisis sistemático, global y específico del yacimiento

3. Analizado los criterios que deben cumplir las empresas mineras durante la selección de sus métodos de minado se determinó que solo el $16.33 \%$, cumplen parcialmente con este modelo de selección de métodos de minado. Por consiguiente se valida la hipótesis planteada en nuestra investigación. Siendo estas empresas las siguientes: Compañía minera San Ignacio de Morococha, unidad San Vicente (Mediana minería); Volcan Compañía Minera S.A.A,unidad Ticlio (Gran minería); Minera Argentum S.A., Morococha (Mediana minería); Minera Argentum S.A, unidad Manuelita (Mediana minería); Minera Argentum S.A., Anticona (Mediana minería); Volcan Compañía Minera S.A.A, unidad Carahuacra (Gran minería); ); Volcan Compañía Minera S.A.A, unidad San Cristóbal (Gran minería); Volcan Compañía Minera S.A.A, unidad San Andaychagua (Gran minería).

4. Analizando la toma de decisiones de las empresas seleccionadas podemos concluir que solo el $5 \%$, realiza una simulación y cuenta con un proceso de diseño capaz de analizar alternativas. Ya que para obtener calidad en el diseño se tiene que simular cada proceso que participa en la obtención de la producción requerida en el minado del yacimiento.

5. El método de asignación de peso mediante un proceso de toma de decisiones junto con el método para la decisión multicriterio. Queriendo plantear un problema de decisión basado en información cuantitativa el cual puede ser evaluado mediante el criterio de expertos. Para resolver este problema se genera una propuesta metodológica la cual consta de las siguientes etapas: 1. Definir el problema 2. Análisis del problema, 3. Búsqueda de alternativas de solución, 4. Decisión en función a criterios de diseño, 5. Especificación de la solución final.

\section{REFERENCIAS BIBLIOGRAFICAS:}

1. Anales de congresos nacionales de Ing. de Minas "Trabajos técnicos de Ing. de Minas". Colegio de Ingenieros del Perú. Lima Perú. 2012.

2. Anales de convenciones de Ing. de Minas "Trabajos técnicos de Ing. de Minas". Instituto de Ingenieros de Minas del Perú. Lima Perú. 2012. 
3. Azadeh, A., Osanloo, M., \& Ataei, M. (2010). A new approach tomining method selection based on modifying the nicholas technique.Applied Soft Computing Journal, 10(4), 1040-1061.

4. Barba Romero, S., \& Pomerol, J., Decisiones Multicriterio.Fundamentos Teóricos y Utilización Práctica., Universidad de Alcalá de Henares, 1997

5. Bitarafan, M.R., Ataei, M., 2004. Mining method selection by multiple criteria decision making tools. J. S. Afr. Inst. Min. Metall.104, 493-498.

6. Bogdanovic, D., Nikolic, D., \& Ivana, I. (2012). Mining methodselection by integrated AHP and PROMETHEE method. Anais DaAcademia Brasileira De Ciencias, 84(1), 219-233.

7. Boshkov, S.H., Wright, F.D., 1973 (Basic and parametric criteria inthe selection, design and development of underground mining systems). SME Mining Engineering Handbook. SME-AIME, NewYork.

8. Cortés Aldana, F. A, García Melón, M., Aragonés, P., Selección de una tecnología de banda ancha para la Universidad Nacional deColombia, sede Bogotá, usando una técnica de decisión multicriterio, Revista Ingeniería e Investigación, Vol.27 No.1, 2007, pp. 132-137.

9. D.E. Nicholas, Selection Procedure, SME Mining EngineeringHandbook, second edition, Society for Mining Engineering,Metallurgy and Exploration, Inc., 1992, pp. 2090-2106.

10. D.H. Laubscher, Selection of Mass Underground Mining Methods,Design and Operation of Caving and Sublevel Stoping Mines, D.Stewart, SME-AIME, New York, 1981 (Chapter 3, pp. 23-38).
11. Figueira, J., Greco, S., and Ehrgott, M., (Eds.) Multiple CriteriaDecision Analysis: State of the Art Surveys, Springer, New York,2005

12. Gómez-Senent, E.; Chiner, M.; Chiner, M.J.,1991. PRES: Programade Evaluación de Proyectos Sociales. VII Congreso Nacional de Ingeniería de Proyectos. Zaragoza 26-28 Junio; pp.27-35

13. Hartman, H.L., 1987. Introductory Mining Engineering. John Wiley,New Jersey.

14. Hartman, H.L., Mutmansky, J.M., 2002. Introductory MiningEngineering. John Wiley, New Jersey.

15. Henig, M.I. and Buchanan, J., Solving MCDM problems: Process concepts, J. Multi-Crit. Decis Anal, 5, 1996, pp. 3-21.

16. Karadogan, A., Kahriman, A., \& Ozer, U. (2008). Application of fuzzy set theory in the selection of underground mining method.Journal of the South African Institute of Mining and Metallurgy,108(2), 73-79.

17. Manual de Minería, estudios mineros del Perú S.A.C. Disponible en: http://www.estudiosmineros. com/ManualMineria/Manual_Mineria.pdf

18. Mendiola Ochante, Víctor, "Manual de Casos de Métodos de Explotación Subterránea I", Huancayo, 2012, 410 pp.

19. Mendiola Ochante, Víctor, "Manual de Casos de Métodos de Explotación Subterránea II", Huancayo, 2011, 410 pp.

20. Universidad Nacional del Altiplano "Explotación Subterránea - Métodos y casos prácticos", Instituto de Ingenieros de Minas del Perú, Facultad de Ing. de Minas - Puno, 1999, 257 p.p. 